\title{
Papel de la oxigenoterapia hiperbárica en el tratamiento de las complicaciones crónicas derivadas del tratamiento con radioterapia en pacientes con cáncer. Bases físicas, técnicas y clínicas
}

\author{
M. Caeiro Muñoz*, A. Calderón González*, A. Mojón Ojea** M. L. López Lóuzara*, \\ J. R. Fernández Bernárdez**, I. Alonso Alonso**, M. L. Vázquez de la Torre*, C. Rodríguez Cerdeira*, \\ V. M. Muñoz Garzón*, R. C. Hermida Domínguez**
}

\section{Resumen}

El incremento de las indicaciones de la Radioterapia (RT) como tratamiento de primera línea en determinados procesos oncológicos, la necesidad de aumentar la intensidad de dosis para un adecuado control local y una mayor sensibilización social del valor de la Calidad de Vida de los pacientes, han impulsado la búsqueda de tratamientos de soporte para prevenir y tratar sus complicaciones de una forma más eficaz y a un coste razonable.

La Oxigenoterapia Hiperbárica (OHB), técnica introducida hace unos 80 años en el tratamiento de los "accidentes de buceo", también ha sido utilizada en procesos relacionados con isquemia/necrosis. Estos antecedentes han supuesto que los investigadores y clínicos que tratamos a los pacientes con cáncer, hayamos abierto una línea de trabajo de la cual podría beneficiarse un grupo de pacientes seleccionado.

En esta revisión describimos las bases físicas, técnicas y fisiopatológicas en relación con la Radioterapia (RT). Además hacemos una revisión básica de las indicaciones de la OHB, en función del área anatómica afecta.

Palabras clave:

Oxigenoterapia Hiperbárica. Radioterapia. Toxicidad Crónica. Cáncer.

Oncología, 2005; 28 (1):20-29

* Hospital do Meixoeiro

** Unidad de Cronobiología. Departamento de Teoría de la Señal y Telecomunicaciones. Escuela Superior de Ingenieros en Telecomunicaciones de la Universidad de Vigo 


\section{Summary}

The increasing indications of radiation therapy (RT) as treatment of choice in specific oncological processes, the need to increase the intensity for an adequate tumor control, and a greater social sensitivity about the value of the patients quality of life, impelled the search of supportive treatments to prevent and treat the radiotherapy complications in a more effective manner, and at a reasonable cost.

The technique of the hyperbaric oxygen ( $\mathrm{HBO}$ ) therapy was introduced 80 years ago for the treatment of diving accidents, and has also been used in processes related to ischemia/necrosis. These background drove the researchers and clinicians treating cancer patients to open a working line that could benefit a group of selected patients.

We describe in this review the physical, technical and physiological bases of radiation therapy (RT). We also make a basic review of the indications of HBO therapy according to the affected anatomical area.

Key words: Oxygen therapy. Hyperbaric. Radiation therapy. Late toxicity. Cancer.

\section{Introducción}

La Oxigenoterapia Hiperbárica (OHB) es una modalidad terapéutica no invasiva, que se fundamenta en la obtención de presiones parciales de oxígeno elevadas, al respirar oxígeno puro en el interior de una cámara hiperbárica a una presión superior a la atmosférical, 2 .

La terapia está diseñada en primer lugar para hacer llegar el suministro de oxígeno a tejidos isquémicos ó enfermos que responden al aumento de los niveles de oxígeno. El segundo efecto básico ó mecánico del oxigeno hiperbárico en el cuerpo humano es el de disminuir el tamaño de la burbuja de tipo cilíndrico y circular, en los accidentes de buceo, donde los niveles de nitrógeno aumentan, ya sea por exposición fuera de los límites ó al ascenso muy rápido a la superficie. Se trata pues de una terapéutica farmacológica, cuyo margen de aplicación está determinado por la presión máxima alcanzada, la duración de la inhalación y la frecuencia y número total de exposiciones.

La terapia es efectiva en un número importante de patologías, con una relación coste/ beneficio adecuada. En los últimos años se ha desarrollado su uso en el campo de la Oncología, en especial, en el tratamiento y prevención de las complicaciones derivadas del uso de la radioterapia (RT).

\section{Historia $^{1,2}$}

La OHB es una tecnología conocida desde hace más de 300 años, cuando se establecieron las leyes de compresión de los gases de 1676 (Ley de Boyle-Mariotte), aunque sólo se utiliza con propiedad desde hace aproximadamente 30 años. Los documentos y testimonios anteriores a 1961 tienen solamente valor histórico o anecdótico. En la segunda mitad del siglo pasado en Europa y en el primer cuarto de siglo en los EEUU, la OHB alcanzó una gran difusión aunque no siempre con suficiente rigor científico.

El primer uso documentado de cámara hiperbárica es anterior al descubrimiento del oxígeno. El médico británico Henshaw parece haber usado aire comprimido con preparados médicos en 1662. Los datos de presión barométrica con oxígeno, como tal, datan de 1775, después de que Priestley descubriera e identificara el oxígeno. En 1834 Junod, construyó una cámara para tratar afecciones pulmonares. En 1837 Pravaz desarrolló una cámara hiperbárica para tratar hasta 50 pacientes. La primera cámara construida en el continente americano fue en Oshawa, (Canadá) en 1860. En 1921 Cuninghan usó la presión parcial elevada de oxígeno para tratar estados hipóxicos. En 1917, Drager en Alemania diseño un sistema para tratar los accidentes buceo. En 1937 Behenke y Shaw, utilizaron oxigeno hiperbárico, para el tratamiento de los accidentes por descompresión. Boerema, en Amsterdam en 1959, relacionó por primera vez el posible efecto terapéutico de las altas concentraciones plasmáticas de oxígeno con las enfermedades causadas por gérmenes anaeróbicos. Fue Gray en 1953 quien introdujo la aplicación de la HBO dentro de la Radioterapia uti- 
lizando un modelo tumoral animal. Pero fue ChurchillDavidson quien por primera vez usó una cámara hiperbárica en el tratamiento de pacientes con Radioterapia.

En los últimos años, tras un periodo de publicaciones de bajo rigor científico que llevaron a la puesta en duda de la eficacia de la técnica, han aparecido publicaciones en campos muy diversos que han permitido volver a considerar su aplicación en áreas tan complejas como la Oncología.

\section{Bases técnicas, físicas, y fisiopatológicas ${ }^{1-4}$}

\section{A. Bases técnicas (Tipos Cámaras)}

\section{A1. Cámaras Monoplaza}

Son de pequeño volumen y para un solo paciente por sesión. Estas son presurizadas con oxigeno puro. La mayoría de estas cámaras constan de amplias estructuras de acero y acrílicos, resistentes a las presiones necesarias para efectuar los tratamientos que usualmente son de tres atmósferas absolutas de presión (ATA) como máximo. Los pacientes son vigilados constantemente (vídeo-cámaras, audio). Debido a las altas concentraciones de oxígeno en éste tipo de cámaras, hay un mayor riesgo de deflagración.

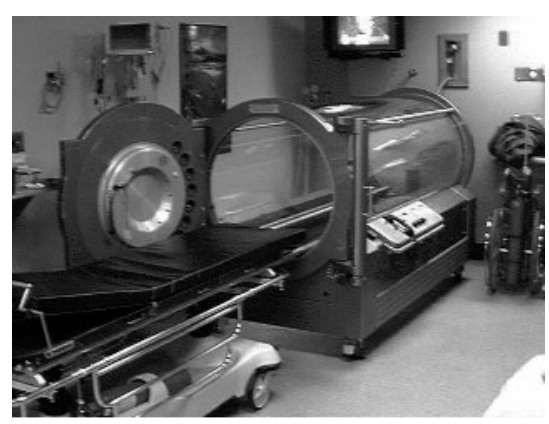

Fig. 1. Cámara monoplaza.

\section{A2. Cámaras Multiplaza}

Estas cámaras son presurizadas con aire comprimido y pueden alojar varios pacientes a la vez. Éstos son provistos de escafandras ó nasofaciales herméticos para recibir oxígeno. Él oxígeno dentro de la cámara se mantiene muy cerca de los valores atmosféricos y el paciente recibe el oxigeno casi al $100 \%$. En la cámara multiplaza el personal médico y paramédico, pueden entrar con los pacientes en caso de necesidad y realizar las técnicas médicas adecuadas en cada caso.

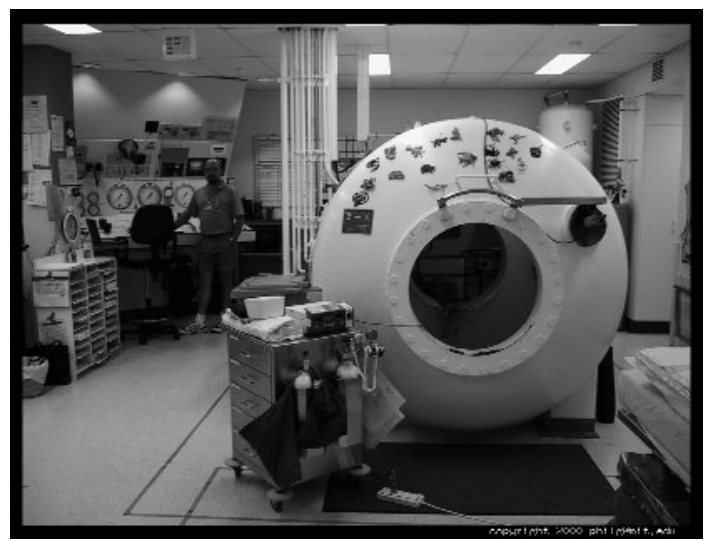

Fig. 2. Cámara multiplaza.

\section{B. Bases Físico-Químicas}

\section{B1. Efecto Volumétrico (Ley de Boyle-Mariotte)}

En virtud de la Ley de Boyle-Mariotte, a temperatura constante el volumen de gas es inversamente proporcional a la presión. Por lo tanto, la elevación de la presión ambiental, disminuye el volumen de las cavidades orgánicas que no están en contacto con las vías respiratorias ${ }^{3,4}$.

\section{B2. Efecto Solumétrico (Ley de Henry)}

Según ésta, al respirar oxigeno en medio hiperbárico se produce un aumento progresivo de la presión arterial de oxígeno que puede superar los $2000 \mathrm{mmHg}$, a un valor ambiental de tres atmósferas absolutas (ATA).

El volumen de oxígeno disuelto y transportado por el plasma mínimo a presión atmosférica aumenta más de 22 veces. De ello se deriva como acción directa, un aumento de la presión venosa de oxígeno, que puede superar los $600 \mathrm{mmHg}$ y de la presión tisular de oxígeno que puede sobrepasar los $400 \mathrm{mmHg}$.

El organismo se protege de la excesiva cantidad de oxígeno produciendo radicales libres oxigenados, sobre cuyo efecto la OHB actúa como modulador, experimentando una vasoconstricción periférica dosis dependiente. A pesar de la disminución del flujo que ésta comporta, la gran hiperoxia logra siempre mantener en todos los casos un saldo de oxígeno favorable, es decir, se trata de una vasoconstricción no hipoxemiante. Estudios realizados por Saltzman en 1968 evidenciaron, en exploraciones fundoscópicas realizadas en medio hiperbárico, que a pesar de la acusada vasoconstricción, la hiperoxigenación era manifiesta. 
Más recientemente Matrhieu y Wattel han confirmado aquellas hipótesis mediante técnicas de capilaroscopia en pacientes con eventos vasculares en curso de tratamiento con $\mathrm{OHB}$.

\section{C) Bases Fisiopatológicas}

La presencia de hiperoxia arterial, venosa, tisular y, sobre todo, el gran aumento del trasporte y disponibilidad del oxígeno plasmático, proporcionan un posible efecto terapéutico en todas las enfermedades en que existía un fenómeno de hipoxia tisular general o local, como factor etiopatogénico preponderante ó bien un cortejo fisiopatológico oxidependiente. La OHB proporciona un aporte adicional transportado por el plasma y no por la masa eritrocitaria. Es un oxigeno que accede por capilaridad a territorios isquémicos terminales y que es transferido a favor de gradiente por difusión simple ${ }^{1-5}$

La OHB produce efectos sobre la angiogénesis, estimulando la microvascularización y la neocolagenización. Su mecanismo de acción está en relación con la facilitación de la hidroxilación de prolina y finalmente la formación de un exuberante tejido de granulación en estados en que por causas hipóxicas ésta se hallaba frenada, por ejemplo, en los tejidos irradiados. Por otro lado la alternancia de hipoxia/normoxia constituye un estímulo angiogénico significativo. Ambos mecanismos están hoy muy bien estudiados y evidenciados con las modernas técnicas iconográficas.

Por mecanismos similares, la OHB ha demostrado también un cierto efecto sobre el metabolismo fosfocálcico, que ha dado lugar a ciertos estudios experimentales tratando de explicar un conocido efecto positivo en los retardos de la calcificación ósea ${ }^{1-5}$.

\section{Efectos de la OHB en relación con las complicaciones de la RT}

Aproximadamente el $50 \%$ de los pacientes diagnosticados de Cáncer llegarán a recibir RT como parte de la estrategia global del tratamiento de su enfermedad. De ellos, un $50 \%$ serán largos supervivientes. Aproximadamente en un 5\% de los pacientes irradiados pueden ocurrir complicaciones severas, las cuales pueden aparecer después de un periodo latente, que varía desde diversos meses, hasta varios años después de administrado el tratamiento radioterápico.

La etiología de los efectos tardíos que la irradiación produce sobre los tejidos sanos está en íntima relación con el efecto oxígeno. La radiación induce daño tisular que afecta al endotelio vascular, causando: hipoxia, progresiva inflamación, fibrosis, isquemia del intersti- cio y subsecuentemente necrosis de tejido, en un periodo de seis meses ó más desde la administración de la misma. Secundariamente la infección compromete frecuentemente al tejido sano, especialmente en presencia de circulación disminuida.

La aparición de secuelas severas con daño por radioterapia, va acompañada de un cortejo de síntomas, que varían en función del órgano $u$ órganos afectos y que, en algunos casos, pueden comprometer la vida del paciente, pero que, en una gran mayoría, producen un empeoramiento de su calidad de vida.

\section{Papel de la OHB en las complicaciones derivadas de la RT según su localización}

\section{Area de Cabeza y Cuello}

\subsection{Osteorradionecrosis mandibular (ORN)}

La mandíbula es un hueso denso, pobremente vascularizado y particularmente vulnerable a la radiación en el área de cabeza y cuello. Teniendo en consideración las propiedades del $\mathrm{O} 2$ a alta presión se han realizado estudios clínicos con la OHB como:

- Profilaxis de la Osteorradionecrosis mandibular.

Marx et al. (1985) en un estudio comparativo aleatorizado con 74 pacientes, demostró un beneficio clínico significativo en el grupo de pacientes con $\mathrm{OHB}$, $5,4 \%$ de ORN frente al $29.9 \%$ en el grupo que no ha recibido OHB (7).

- Tratamiento de Osteorradionecrosis mandibular establecida (ORN)

La utilización OHB por encima 2,0 atmósferas de presión (ATA) durante 90-120 minutos, con 02 al $100 \%$ ha dado lugar a los mejores resultados. Esto se ha visto en el trabajo de Marx et al. (1984) con 70 pacientes alcanzando niveles de resolución del $100 \%$ y Tobey et al. (1979) asociado a cirugía agresiva en 12 pacientes, los cuales presentaron significativa mejoría clínica.

Otros estudios de Mainous, Hart, Farmer, Mounsey, Mackenzie, Curi y David han obtenido resultados alentadores. Un único estudio, el de Maier y col. (2000) en 41 pacientes, no ha demostrado beneficio clínico.

Podremos establecer como conclusión la recomendación de utilizar la OHB como tratamiento electivo en la ORN ${ }^{7}, 9$.

\subsection{Radionecrosis de tejidos blandos de cabeza y cuello}

El grueso de los estudios lo constituyen fundamentalmente las necrosis laríngeas: los resultados de las se- 
ries de Davis (1979), Ferguson (1987), Feldmeier (1993), Filintisis (2000), Narozny (2001) y, sobre todo, de Marx (1999) con 160 pacientes (estudio comparativo no randomizado), demuestran que existe una mejoría clínica en todas las series analizadas.

Deberemos concluir diciendo que la $\mathrm{OHB}$ es una terapéutica electiva en la radionecrosis de los tejidos blandos en el área en cabeza y cuello, en especial, en la Osterradionecrosis de mandíbula y la necrosis laríngea $^{7}$.

1.3 Otras indicaciones, como los "Implantes dentales" han sido introducidas recientemente, realizándose en pacientes previamente irradiados; 0 a los que se les va a realizar previamente una extracción dentaría ${ }^{31}$.

\section{Area del Sistema Nervioso Central (SNC) y Sistema Nervioso Periférico (SNP)}

La OHB en relación con las complicaciones del SNC, ha sido estudiada de manera muy limitada. Estas se asocian fundamentalmente a técnicas de Radiocirugía Exterotáxica y Braquiterapia Intesticial en el caso del encéfalo y con Radioterapia Convencional en la médula espinal y plexopatía braquial.

\subsection{La OHB en mielopatías (mielitis actínica)}

Glassbarm y otros publicaron una serie con 9 pacientes. En 6 de ellos hubo mejoría con OHB. Angibaud y otros revisaron el papel de la $\mathrm{OHB}$ en las mielopatías postirradiación con resultados razonables $(7,10)$.

\subsection{La OHB en Encefalopatías Actínicas (Necrosis cerebral)}

El papel OHB se ha estudiado en una serie de 10 pacientes pediátricos analizados por Chuba y otros. Los resultados hallados demostraron que la sintomatología neurológica mejoró en los 10 pacientes tratados, de los cuales 8 presentaban necrosis cerebral ${ }^{11}$.

\subsection{La OHB en la toxicidad de los nervios y quiasma ópticos}

Guy y Shotz en 4 pacientes con neuropatia óptica severa demostraron una mejoría significativa en el $50 \%$ de los mismos 7,12 .

Roden y otros, incluyeron un total de 13 pacientes con OHB y corticosteroides. El análisis de los resultados no demostró una mejoría significativa de la asociación en la mayoría de los pacientes ${ }^{7}, 12$.

\subsection{La OHB en la plexopatía braquial inducida por RT} Pritchard y otros, en un estudio fase II aleatorizado a doble ciego con 34 pacientes, observaron que no había diferencias significativas en los tests neurofisiológicos, entre los pacientes que habían recibido $\mathrm{OHB}$ frente a los pacientes que habían recibido una mezcla de aire a presión atmosférica. Pero si se vio un importante retardo en la aparición de los síntomas en el grupo tratado con OHB, frente al grupo control. Los 6 sujetos investigados en los que coexistía linfedema crónico del brazo, mejoraron del mismo.

En conclusión y con los datos actuales, la OHB no ha demostrado un claro beneficio clínico, aunque se tendría que profundizar en la investigación, ya que algún subgrupo de pacientes podría beneficiarse ${ }^{13}$.

\section{Area Torácica (Pared torácica y Mamas)}

\subsection{Pared torácica}

Feldmeier y otros realizan una revisión retrospectiva de 23 casos, que habían sido tratados con OHB como terapia adyuvante para la necrosis radio-inducida de la pared torácica. En 8 pacientes, la afectación era únicamente de tejido blando y 15 tenían asociada necrosis de hueso y necrosis de tejido blando. Cuando sólo el tejido blando estaba afectado, la asociación de desbridamiento no quirúrgico y $\mathrm{OHB}$ fue altamente efectiva. Todos los pacientes de este grupo que no tenían enfermedad, resolvieron su necrosis. Del grupo de pacientes que tenían necrosis de tejido blando y hueso, un total de 8 de los 15 pacientes habían resuelto su daño radioterápico. De los 6 que fallaron, en 3 no se había realizado una resección quirúrgica, y en dos se había realizado desbridamiento agresivo. Los autores establecieron como recomendación:

- las necrosis de tejidos blandos de la pared costal se pueden tratar con OHB de manera exclusiva.

- los pacientes que llevan asociado necrosis de hueso (costillas, esternón) es necesario realizar desbridamiento quirúrgico para extirpar todo el hueso necróti$\mathrm{co}^{14}$.

\subsection{Mama}

El tratamiento conservador de la mama es el tratamiento estándar para la mayoría de los estadios I y II del cáncer de mama. Un porcentaje no despreciable de casos presenta secuelas tardías consistentes en ede$\mathrm{ma}$, dolor y cambios en la piel.

Basándose en series con casos aislados, Carl y otros iniciaron un estudio comparativo no aleatorizado con 44 pacientes, de los que 32 recibieron $\mathrm{OHB}$ en cámara multiplaza, con una mediana de 25 sesiones (7-60 de rango). El 02 se administraba al 100\% a 2,4 ATA, en sesiones de 90 minutos, 5 veces por semana. El grupo control consistía en 12 pacientes que rechaza- 
ron el tratamiento con $\mathrm{OHB}$. Se observó una significativa reducción de la sintomatología (dolor, edema y eritema), comparado con el grupo control $(p<0,001)$. En 7 de las 32 mujeres no hubo sintomatología, frente a las 12 restantes del grupo control que permanecieron sintomáticas ${ }^{15}$.

\section{Area abdomino-pélvica}

\subsection{La OHB en la enteritis rádica}

La radioterapia es usualmente aplicada como tratamiento complementario ó primario de los cánceres pancreáticos, tracto biliar, gástrico y enfermedades del colon. Se han observado en esta localización hasta un $19 \%$ de complicaciones serias. Estas suelen aparecer a partir de los 6 meses y típicamente son el resultado de la hipoxia secundaria que reactiva el proceso de fibrosis - endarteritis, que se desarrollan en el estroma de los tejiidos irradiados.

El tratamiento de las complicaciones, en su forma más severa, a menudo requiere intervención quirúrgica. Muchas complicaciones pueden también suponer o significar la disminución de la calidad de vida de los supervivientes por cáncer.

Feldmeier y otros realizaron un estudio retrospectivo en un total de 44 pacientes que fueron tratados desde 1979, de los cuales 41 pacientes fueron analizados. Las localizaciones afectadas fueron pared abdominal, intestino grueso, intestino delgado y huesos pélvicos. Se observó que un total de 26 pacientes habían curado, 6 fallaron a la curación y 9 tuvieron un inadecuado ciclo de tratamiento. Especialmente interesante fue la resolución de fístulas en 6 de los 8 pacientes, de las cuales sólo 3 requirieron cirugía para su cierre ${ }^{16}$.

Gouëllo y otros realizaron un estudio retrospectivo en 36 pacientes con necrosis de tracto digestivo (enteritis rádica, proctitis rádica o abscesos perianales). En 21 pacientes $(66 \%)$ hubo beneficio clínico. A corto plazo 19 pacientes (53\%) mejoraron y $17(47 \%)$ fallaron. A largo plazo un total de 32 pacientes fueron evaluados, de éstos 9 pacientes experimentaron cura de toda su sintomatología y 12 pacientes experimentaron mejoría significativa a largo plazo. Los otros 11 (34\%) fallaron ${ }^{17}$.

Nakada y otros, publicaron un caso de enteritis rádica en intestino grueso (colon sigmoide), con importante sangrado y hallazgos endoscópicos e histológicos característicos. La administración de OHB fue seguida de una desaparición del sangrado digestivo y reversión de los hallazgos endoscópicos y anatomopatológicos ${ }^{18}$.

La experiencia en tratamientos combinados para la reparación de secuelas de tratamientos con radiotera- pia abdómino - pélvica es muy limitada. Brandon y colaboradores en un intento por mejorar los resultados de la cirugía, la asociaron con la administración de $\mathrm{OHB}$. Un total de 5 casos, con pacientes que habían presentado graves secuelas en tejidos blandos tras haber sido tratados con RT y que estaban programados para una intervención quirúrgica abdominal, fueron sometidos previamente a $\mathrm{OHB}$ con una cámara monoplaza. El resultado final fue que los pacientes experimentaron una mejoría clínica significativa de los síntomas asociados a las complicaciones digestivas y urológicas, que acompañan a la RT abdominal y pélvica ${ }^{19}$.

Feldmeier y otros realizaron un estudio en ratones $\mathrm{C} 3 \mathrm{H}$, para determinar el papel de la OHB administrada para prevenir la enteropatía rádica crónica. Un total de 50 ratones divididos en dos grupos, recibieron 30 Gy de irradiación abdomino-pélvica en 10 fracciones. El grupo de estudio recibió un ciclo de 30 sesiones de OHB iniciados 7 semanas después de la exposición a la radiación. El grupo control recibió únicamente soporte nutricional y cuidados ambulatorios. Un tercer grupo de tres animales no recibió irradiación ó HBO. Todos los animales fueron sacrificados 7 meses después de la radiación. Se realizó una inspección grosera para objetivar la enteropatía. Se utilizó un sistema de medida para cuantificar la estrechez y rigidez del ileon cercano a la válvula ileocecal. Los animales que recibieron $\mathrm{OHB}$ tenían pocos signos de enteropatía actínica y se vio que tenían menos estrechez y menos rigidez en los segmentos intestinales recolectados que los que no la recibieron, siendo los hallazgos estadísticamente significativos ${ }^{20}$.

\subsection{La OHB a nivel ano-rectal}

A demás de los trabajos de Feldmeier ${ }^{16}$ y Gouëllo ${ }^{17}$ que cuantificaron el grado de severidad de los síntomas y hallazgos anatomapatológicos a través de una escala, la mayoría de publicaciones son series de casos muy heterogéneas, tanto en los criterios de inclusión, como en el método de administración de la $\mathrm{OHB}^{8}$. Warren y colaboradores publicaron en 1997 una serie de 14 pacientes con proctitis crónica radioinducida. Nueve pacientes fueron tratados con $\mathrm{OHB}$ en cámara monoplaza de 2 atmósferas (2 ATA) y 5 pacientes fueron tratados con OHB a 2'36 ATA. Los resultados mostraron que 8 pacientes experimentaron una completa desaparición de los síntomas y que un paciente tenía una sustancial mejoría, lo que constituyó un total de respuestas del $64 \%$. El rango de seguimiento fue de 5 a 35 meses (mediana de 17). Un total de 5 pacientes fueron considerados no respondedores. Tres experimentaron significativa mejoría durante el tratamiento pero como recidivaron tan pronto, la tera- 
pia fue discontinuada. Los otros dos no presentaron mejoría en ningún momento. En las sigmoidoscopias practicadas después de la terapia se pudo documentar una mejoría endoscópica frente a los no respondedores que no la presentaban ${ }^{21}$.

Chong Seng Woo y otros publicaron una revisión retrospectiva de 18 pacientes diagnosticados de proctitis rádica tratados con OHB. Los síntomas principales fueron sangrando, diarrea, incontinencia y dolor. En más de la mitad de éstos, los síntomas se resolvieron parcial o totalmente 22 .

Bem y colaboradores, publicaron 2 casos de complicaciones ano-rectales con carcinoma anal relacionados directamente con una anorrectitis actínica. En ambos casos se introdujo la $\mathrm{OHB}$ tras el diagnostico de anorectitis actínica. El modelo de administración de OHB fue de 2,4 ATA, durante 90 minutos, por 5 días a la semana. Los dos pacientes experimentaron mejoría paulatina, estando libres de síntomas a los 3 meses y 4 años del último tratamiento ${ }^{23}$.

Kitta y otros presentan una serie de 4 pacientes con diagnostico de carcinoma de próstata, que padecieron una proctitis rádica crónica y fueron tratados con $\mathrm{OHB}$. Todos los pacientes completaron el tratamiento con $\mathrm{HBO}$ al $100 \%$, en una cámara multiplaza, durante 60 minutos al día y 5 días a la semana. El número de sesiones variaba en función de la respuesta clínica. Un paciente mejoró completamente de su sintomatología de sangrado rectal. Otro paciente mantenía un mínimo sangrado rectal. Otro paciente recidivó de su sangrado rectal a los 3 meses de finalizada la OHB. En el cuarto paciente no hubo mejoría y se mantuvo con severa proctalgia. Podemos afirmar que se obtuvo un beneficio clínico moderado con tratamiento de OHB, no cuantificado estadísticamente ${ }^{24}$.

Jhonston y otros, realizaron una revisión del papel de la $\mathrm{OHB}$ en el tratamiento de las complicaciones tardías de la radioterapia pélvica a nivel del ano y del recto. Los autores llegaron a la conclusión de que la principal causa de este cuadro está en íntima relación con la isquemia, lo que da lugar a una inestable neovascularización, causando sangrado rectal. La aplicación de la OHB produce una reversión del componente fibrótico y estimula la neovascularización de la zona. En su revisión observaron que un $65 \%$ de los pacientes con proctitis rádica severa mejoraron, aunque en un porcentaje de los mismos puede haber recurrencias a largo plazo. En sus recomendaciones finales establecen la necesidad de realizar un ensayo clínico amplio multicéntrico $y$, a ser posible, internacional ${ }^{25}$.

Denton y otros realizaron una revisión sistemática del papel de la $\mathrm{OHB}$ en el contexto general de las intervenciones no quirúrgicas para el manejo de la proc- titis rádica tardía, con vistas a establecer recomendaciones terapéuticas en relación con la metodología de la Medicina Basada en la Evidencia. En su revisión establecieron que hay al menos 9 referencias relevantes, con un total de 86 casos. Un total de 8 series fueron analizadas. Sólo una era un estudio prospectivo, las demás eran series de casos con características heterogéneas. En sólo dos de estos trabajos, Feldmeier (1996) y Gouëllo (1999), tenían desarrollado un sistema de cuantificación del grado de proctitis rádica crónica con una escala que midía el grado de hallazgos histológicos y síntomas. El número de tratamientos depende del grado de la lesión establecida. La cuantificación de la respuesta fue inicialmente hecha con una vaga descripción de la resolución de los síntomas y no mediante un análisis estadístico reglado. La duración de la respuesta fue recogida de manera inconsistente y los datos ó escalas de calidad de vida, no se recogieron en ningún informe. Los efectos secundarios fueron publicados en ocho, todos ellos en relación con barotrauma acústico de carácter leve.

Las conclusiones finales de esta revisión, a pesar de que aparentemente la $\mathrm{OHB}$ puede influir en los cambios secundarios a la radiación en el intestino grueso que son refractarios a otros tratamientos, el grado de beneficio clínico, así como la duración de la respuesta no han sido cuantificados, tanto por la metodología utilizada como por la falta de escalas de calidad de vi$\mathrm{da}^{8,26}$.

\subsection{La OHB en el tratamiento de las cistitis \\ hemorrágicas causadas por RT}

Las cistitis hemorrágicas están reconocidas como un posible efecto secundario de la RT administrada en un conjunto de enfermedades pélvicas. Los pacientes pueden experimentar síntomas como dolor, sangrado y retención urinaria. Varios tratamientos paliativos han sido establecidos con diferentes resultados. Del Pizzo y otros, trataron con $\mathrm{OHB}$ un total de 11 pacientes con cistitis hemorrágica inducida por radiación. Los síntomas que presentaban eran hematuria y otros asociados, como dolor suprapúbico y urgencia miccional Todos ellos recibieron $\mathrm{O} 2$ al $100 \%$, en cámara multiplaza a 2 ATA, durante 90 minutos por 5 días en una semana. El promedio de tratamiento fue de 40 sesiones (rango, 28-64).En un total de 11 pacientes: 3 (27\%) estaban libres de síntomas y $3(27 \%)$ tenían síntomas persistentes que han requerido derivación urinaria supravesical. Los otros 5 pacientes respondieron inicialmente a la OHB pero presentaron recurrencia a largo plazo y han necesitado otras terapias definitivas ${ }^{27}$.

Mathews y otros, revisaron un total de 17 pacientes con cistitis hemorrágica que habían recibido $\mathrm{OHB}$ tras 
fracaso de otras modalidades terapeuticas estándar. La OHB fue administrada una única vez al día (media de 21). La hematuria se resolvió en $11 / 17(64 \%), 2$ tenían sólo hematuria macroscópica y 2 tenían únicamente hematuria microscópica residual, 3 pacientes habían mejorado pero murieron precozmente de complicaciones relacionadas con el cáncer tras finalizar el tratamiento con $\mathrm{OHB}$ y 2 tenían recurrencia de hematuria macroscópica ó franca. La aplicación precoz de $\mathrm{OHB}$ estaba asociada con una rápida resolución de la cistitis hemorrágica ${ }^{28}$.

\subsection{La OHB en el tratamiento de las complicaciones de la RT en los tejidos ginecológicos}

Williams y colaboradores, vieron en 14 pacientes que presentaban necrosis de tejidos blandos y que habían fallado a tratamiento conservador, al menos tres meses después de finalizado éste. La técnica empleada fue una cámara monoplaza a 2,0 ATA, con una concentración de $\mathrm{O} 2$ al $100 \%$. Recibieron un total de 15 tratamientos. Las pacientes que tenían radionecrosis vaginal aislada ó en asociación con fístula recto-vaginal alcanzaron una completa resolución de la necrosis con OHB. Unicamente una paciente presentó fallo al tratamiento. En este estudio se demostró un beneficio clínico de la $\mathrm{OHB}^{29}$.

\section{La OHB en el tratamiento de las complicaciones secundarias de la RT en las extremidades}

Feldmeier y colaboradores, revisaron retrospectivamente 17 pacientes ( 9 mujeres y 8 hombres), tratados entre 1979 y 1997 por radionecrosis de las extremidades dentro de los campos previamente irradiados por diferentes patologías malignas, en su mayor parte, sarcoma de tejidos blandos seguidos de cánceres de piel. La OHB fue administrada mediante una cámara multiplaza a 2,4 ATA, administrados diariamente, por 90 minutos al $100 \%$ de presión de oxigeno. Un total de 11 pacientes (65\%) sanaron completamente, mientras 5 (29\%) no mejoraron y en $1(6 \%)$ se perdió el seguimiento. En $3(60 \%)$ de las pacientes que fallaron, se vio que tenían recurrencia local y/o a distancia de su tumor de manera precoz, y hubo que discontinuar la administración de OHB. El paciente que se había perdido seguimiento, en su último control había mejorado de los síntomas pero no llegaron a desaparecer los mismos. De los que fallaron, 4 pacientes requirieron amputación (2 pacientes con recurrencia asociada). Si se excluye los pacientes con recurrencia y el paciente perdido de seguimiento, la tasa de mejoría es $11 / 13$ (85\%), que evitaron la amputación. El $80 \%$ de los 5 fallos, requirieron amputación de la extremidad afecta ${ }^{30}$.

\section{Conclusiones}

Podemos afirmar que la $\mathrm{OHB}$ es una técnica segura, fácil de administrar, con tasas de efectividad que la podrían convertir en una técnica estándar en las complicaciones crónicas de la RT de determinadas localizaciones anatómicas.

El nivel de evidencia es alto a nivel del área de cabeza y cuello, especialmente en las osteorradionecrosis de mandíbula, el cual puede considerarse tratamiento de elección. A nivel del sistema nervioso ha demostrado un nivel de eficacia elevado en las necrosis cerebrales, siendo mucho menor en las mielopatías y pares craneales aunque se ha podido demostrar beneficio clínico en un número importante de pacientes. En nervio periférico (plexo braquial) no hemos podido demostrar un claro beneficio clínico como tratamiento paliativo, pero podría tener un papel más destacado como tratamiento profiláctico.

En las complicaciones severas localizadas en la pared torácica, la $\mathrm{OHB}$ con o sin cirugía pueden resolver el daño de la RT en mayor ó menor grado en función del grado necrosis ósea asociado. En la mama, existe un claro beneficio en la mayor parte de los síntomas asociados (edema, dolor, eritema) pero no se demostró mejoría en los síntomas dependientes de la plexopatía braquial.

En la localización abdominal, tanto en la enteropatía, proctitis, cistitis, fístulas recto-vaginales, vesico-vaginales, etc, los diferentes estudios analizados han concluido que puede ser una técnica alternativa a otras tanto de carácter conservador como de carácter invasivo. En algunas ocasiones esta técnica puede complementar a otras, en especial a la cirugía cuando se administra de manera profiláctica.

Otro grupo de complicaciones son las localizadas en las extremidades tratadas con RT. En éstas se ha podido ver que hay un beneficio importante cuando va asociada a control de enfermedad, pero si hay enfermedad persistente o recurrencia va acompañada de un alto porcentaje de fracaso que muy posiblemente acaben en amputación de la extremidad.

Como recomendación final y dada la dificultad práctica de incluir a estos pacientes dentro de ensayos clínicos controlados, podríamos considerar la disponibilidad de esta arma terapéutica en nuestro arsenal de tratamientos paliativos reparadores y con ello contribuir a la mejora de su calidad de vida. 


\section{Bibliografía}

1. M Aboud Aoun, Medicina Hiperbárica, Sociedad Panameña de Pediatría. Disponible en: http//www.spponline.net/artículos/art_mhiperbarica.ntm/

2. L Paz Valiñas, Novas Tecnoloxías, Redecubriendo a oxigenoterapia hiperbárica. Revista Galega de Actualidade Sanitaria, vol. 2, (4), 318-322. Decembro 2003.

3. Desola J. Bases y Fundamentos Terapeuticos de la oxigenoterapia hiperbárica, JANO/Medicina 1998 LIV (1260) 5-11.

4. Desola J. Indicaciones y contraindicaciones de la oxigenoterapia hiperbárica. JANO/Medicina 1998; LIV (1260).

5. Halley D. Hyperbaric oxygen therapy-recent finding 0 evidence for its effectiveness. Alberta Heritage, Foundation for Medical Research 2003

6. Hyperbaric oxygen in the treatment of radio-induced lesions in normal tissues. I Fith European Consensus Conference on Hyperbaric Medicina Lisbon; 2001.

7. Feldmeier JJ and Hampson NB. A systematic review of the literature reporting the application of hyperbaric oxygen prevention and treatment of delayed radiation injures: An evidence based approach. Undersea and Hyper Med 2002;22(1); 4-28.

8. Denton A, Forbes A, Andreyevd J, Maher EJ. Interventions for late radiation proctitis in patients, who have received radical radiotherapy to the pelvis (Cochrane Review): In the Cochrane Library Issue 1, 2004, Chichester, UK: John Wiley y D Sons, LTD.

9. Wersford-Brown CE and N.B. Hampson. Hyperbaric oxygen treatment protocols for mandibular osteoradionecrosis. Undersea Hyperb. Med. 2003; 30 (3) 175179.

10. Angibaud, G Ducasse JL, Baille G, Clanet M. Potential value of hyperbaric oxygenation in the treatment of postradiation myelopathies (abstradd). Rev Neurol. (Paris ) 1995 Nov; 151(11): 661-666.

11. Chuba P, Aronin P. Hyperbaric oxygen therapy for radiation-induced brain injury in children. Cancer 1997; 80: 2005-2012.

12. Roden D, Bosley TM Fowble. Delayed Radiation Injury the retrobulbar optic nerves and chiasm. Clinical syndrome and treatment with hyperbaric oxygen and corticosteroids. Ofthalmology 1990; 97: 346-351.

13. Pritchard, Anand P, Broome J. Double-blind randomized phase II study of hyperbaric oxygen in patients with radiation induced braquial plexopathye. Radiothe. Oncol. $2001 ; 58:$ 279-286.

14. Feldmeier JJ, Heimbach RD, Davolt DA, Court WS, Stegmann BJ and Sheffield PJ. Hyperbaric Oxygen as an adjunctive treatment for delayed radiation injury of the chest wall: A retrospective review of twenty tree cases. Undersea Hyperbaric Med 1995; 22(4) 383-392.

15. Carl UM, Feldmeier JJ, Schmitt G, Hartmann KA.. Hy- perbaric Oxygen Therapy for late sequel in women receiving radiation after breast-conserving surgery. In J Radiat Oncol Biol Phys 2004; 29 (4) 1029-1031.

16. Feldmeier JJ. Heimbach RD, Davolt DA, Court WS, Stegmann BJ, Sheffield PJ. Hyperbaric oxygen an adjunctive treatment for delayed radiation injuries of the abdomen and pelvis. Undersea Hyperbaric Med (1996; 23, (4) 205-213.

17. Gouëllo JP, G Bouachour, B Person, J Ronceray, P Cellier, Ph. Alquier. Intérêt de l'oxygènothérapye hyperbase dans la pathologíe digestive post-radique Press Med 1999; 28 (20):1053-1057.

18. Nakada T, Kubota Y, Sasagawa I. Therapeutic Experience of Hyperbaric Oxygenation in Radiation Colitis: Report of a case. Dis Colon Rectum 1993; 36: 962-965.

19. Pomeroy BD, Keim LW, Taylor RJ. Preoperative Hyperbaric Oxygen Therapy for radiation induced injures. J. Urol 1998; 159 (5): 1630-1632.

20. Feldmeier JJ, Jelen I, Davolt DA, Valente, PT, Meltz ML Alecu R. Hyperbaric oxygen as a prophylaxis for radiation-induced delayed enteropaty. Radiotherapy and Oncology 35 (1995) 138-144.

21. Warren DC, Feehan P, Slade JB, Cianci PE.. Chronic radiation proctitis treated with hyperbaric oxygen. Undersea Hyperbaric Med. 1997 Sept; 24 (3): 181-4.

22. Woo TC, Joseph D, Oxer H. Hyperbaric Oxygen Treatment for radiation proctitis. Int. J. Radiation. Oncology. Biol. Phys 1997: 38 (3) 619-622.

23. Bem J, Bem S, Singh A,. Use of Hyperbaric Oxygen Chamber in the manegement of radiation. Related complications of the anorectal region report of two cases and review of the literature. Dis Colom Rectum 2000; 43: 1435-1438.

24. Kitta T, Shinohara N, Shirato H, Otsuka H, Koyanagi T. The treatment of chronic radiation proctitis with hyperbaric oxygen in patients with prostate cancer. BJU Int, 2000: 85(3):372-374.

25. Jhonston MJ, Robertson GM, Frizelle FA. Management of late complications of pelvic radiation on the rectum and anus: A review Dis Colon Rectum 2003 46: 247259.

26. Denton AS, Andreyev HJN, Forbes A, and EJ Maher. Systematic review for non-surgical intervention for the management of late radiation proctitis. BJC 2002; 87; 134-143.

27. Del Pizzo JJ, Chew BH, Jacobs SC, Sklar GN. Treatment of radiation induced hemorrhagic cystitis with Hyperbaric Oxygen: Long Term Follow-up. J. Urol, 1998; 160 (3): 731-733.

28. R Mathews, Rajan N, Josefson L Camporesi E, and Makhuli Z. Hyperbaric Oxygen Therapy for radiation induced hemorrhagic cystitis. J. Urol 1999; 161: 435-437.

29. Williams JA, Dick Clarke, Dennis WA, Dennis EJ 3ed, Smith ST. The treatment of pelvic soft tissue radiation necrosis with hyperbaric oxygen. Am J Obstet Gynecol 1992; 167:412-416. 
30. Feldmeier JJ, Heimbarch D, Davolt et al. Hyperbaric oxygen in the treatment of delayed radiation injures of the extremities. Undersea Hyper Med 2000; 27(1) 1519.
31. Pasquier D, Hoelscher T, Schmutz, Dische S, et al. Hyperbaric oxygen therapy in the treatment of radio-induced lesions in normal tissues: a literature review. Radiotherapy and Oncology 72 (2004) 1-13.

Correspondencia:

Dr. M Caeiro Muñoz

O Fuxon, 14

Tirán-Moaña

E-36958 Pontevedra

E-mail: caeiro.munoz@cmpont.es 